Muhammad Hossain, Ann Dewey, John Crossland, Rebecca Stores, Yohai Hakak, Shipa Khan

\title{
Perception and attitude towards ageing and dementia among the Bangladeshi community in England
}

Background: Worldwide, an increasing ageing population mean more people may suffer from dementia. Despite a rapid growth of older Bangladeshi immigrants living in the UK, there is little evidence about how community members (who are not affected by dementia) understand and view the process of ageing and dementia. Yet, Bangladeshis have a higher risk of developing type 2 diabetes and heart disease; both important indicators of developing dementia in old age. In previous research, conducted with immigrant South Asians, the concept of stigma and its impact on the understanding of dementia has been commonly acknowledged as a big challenge to seeking service support. This study aimed to explore and analyse the perception, knowledge, and attitude of ageing and dementia among the Bangladeshi community.

Methods: Two separate focus group discussions (one male, $n=12$; and one female, $n=9$ ) were held with Bangladeshi immigrants in a large South coast city. Conducted either in English (male group) or in Bengali (Sylheti dialect) for the female group, a topic guide was used. Two vignettes of early and late dementia were used to stimulate discussion. Data were audio recorded, transcribed and translated into English then thematically analysed using NVivo 10 software.

Results: Perceptions of personal ageing process was mostly related to physical decline. Across the genders, lack of knowledge and understanding of dementia awareness was evident, but stigma about dementia non-existent. In contrast, there was a strong sense of stigma attached to being a paid carer. Respondents felt that current healthcare services failed to meet religious needs of Bangladeshi Muslim older people, and subsequently could hinder or deter Bangladeshi people living with dementia and their carers' willingness to seek and use appropriate health and support services.

Conclusions: This study provides current research findings where no other qualitative research have examined the knowledge and attitude about dementia among a Bangladeshi community in England. In this context, it is vital that future study highlights the importance of raising dementia awareness and provides more information to Bangladeshi community members about dementia, dementia caregiving as well as religiously sensitive healthcare services. 